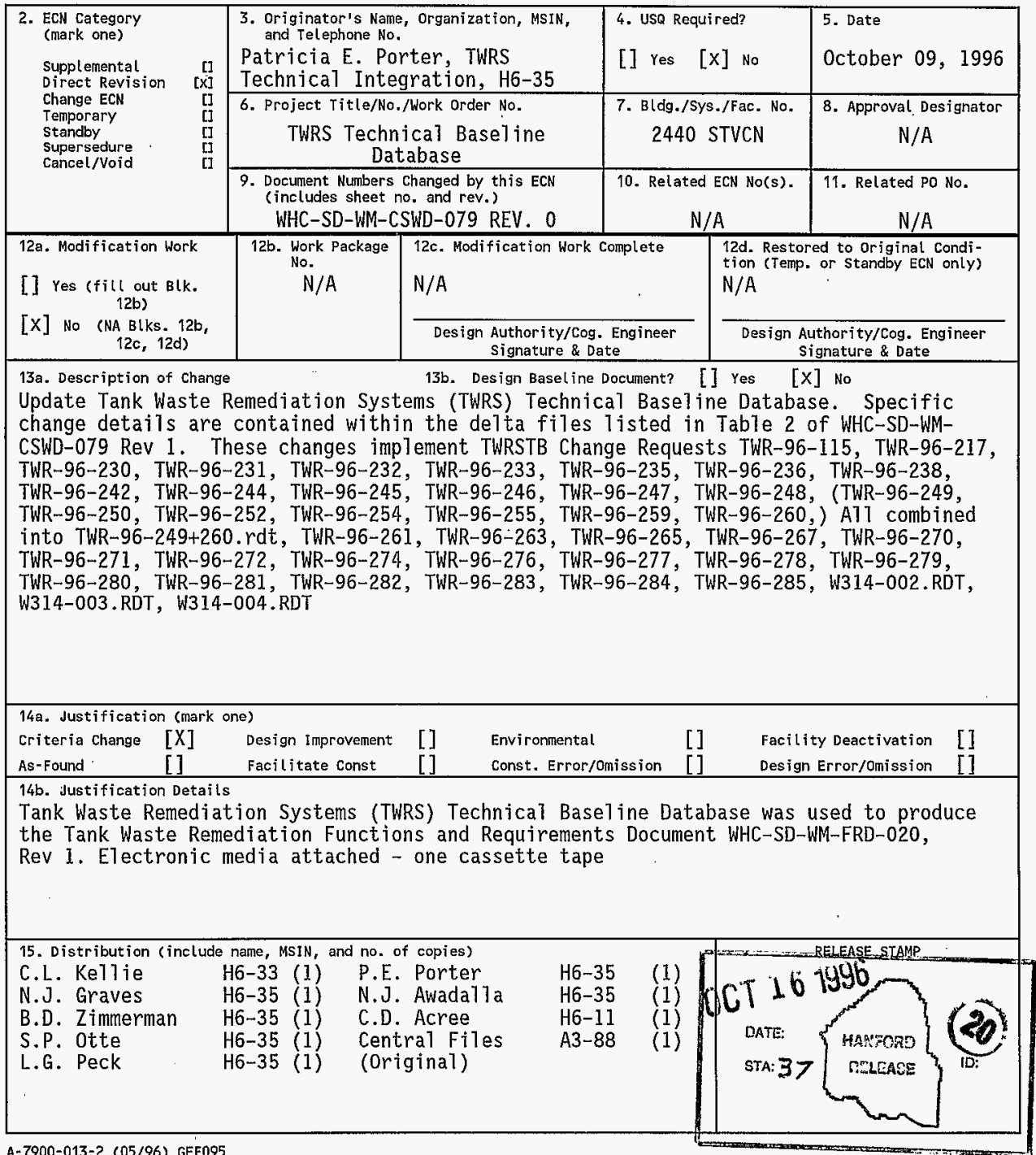




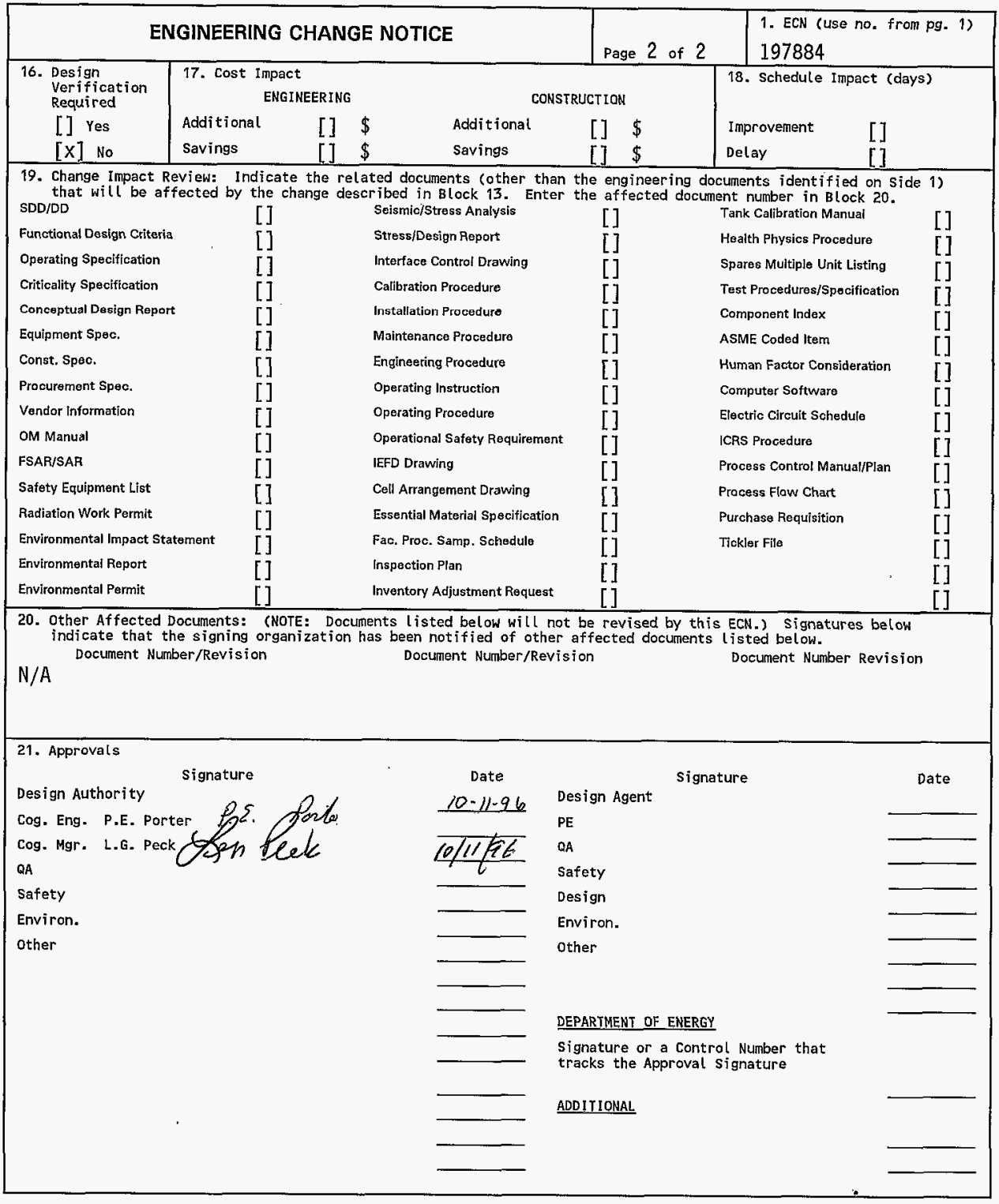




\section{Tank Waste Remediation Systems Technical Baseline Database}

Patricia E. Porter

LMHC, Richland, WA 99352

U.S. Department of Energy Contract DE-AC06-87RL10930

EDT/ECN: 197884

Org Code: 74440

B\&R Code: EW3120075

UC: 905

Charge Code: D1N3A

Total Pages: 14

Key Words: Tank Waste Remediation Systems, Technical Baseline, Tank Waste Remediation Functions and Requirements, RDD-100, Systems

Engineering

Abstract: This document includes a cassette tape that contains Hanford generated data for the Tank Waste Remediation Systems Technical Baseline Database as of October 09, 1996.

TRADEMARK DISCLAIMER. Reference herein to any specific comercial product, process, or service by trade name, trademark, manufacturer, or otherwise, does not necessarily constitute or imiply its endorsement, recomendation, or favoring by the United States Government or any agency thereof or its contractors or subcontractors.

Printed in the United States of America. To obtain copies of this document, contact: WHC/BCS Document Control Services, P.O. Box 1970, Mailstop H6-08, Richland WA 99352, Phone (509) 372-2420; Fax (509) 376-4989.
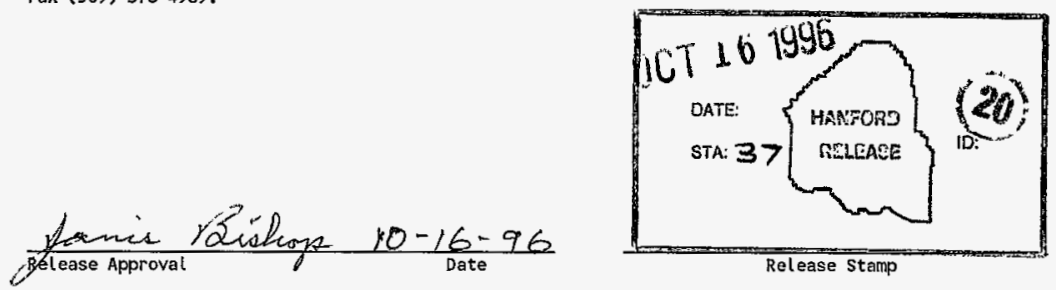

Approved for Public Release 


\section{RECORD OF REVISION}

(2) Title

Tank Waste Remediation Systems Technical Baseline Database

CHANGE CONTROL RECORD

(3) Revision (4) Description of Change - Replace, Add, and Delete Pages

(7) Initial Re]ease, EDT-603661

1 RS Update Tank Waste Remediation Systems

Technical Baseline Database to incorporate

information from continued development activities. See ECN 197884

Authorized for Release

(5) Cog. Engr. (6) Cog. Mgr, Date

\begin{tabular}{l|l|l}
$N / A$ & $N / A$
\end{tabular}

P. Porter

pipontw

$10-11-96$

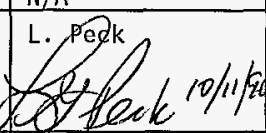




\subsection{PURPOSE AND SCOPE}

The attached cassette tape contains the Hanford specific files (Table 1) that make up the Tank Waste Remediation Systems (TWRS) Technical Baseline Database. The cassette tape also includes the delta files (Table 2) that delineate the development of revision 1 of the TWRS Technical Baseline Database. This information is being managed and maintained on the TWRS RDD100 System, which uses the capabilities of RDD-100, a systems engineering software system of Ascent Logic Corporation (ALC). This revision of the TWRS Technical Baseline Database uses RDD-100 version 4.0.3 (see Table 3).

Directories reflect those controlled by the TWRS RDD-100 System Administrator. Table 4 provides information regarding the platform.

\subsection{RESPONSIBILITIES}

The TWRS Technical Integration organization is responsible for the development and maintenance of this document. 


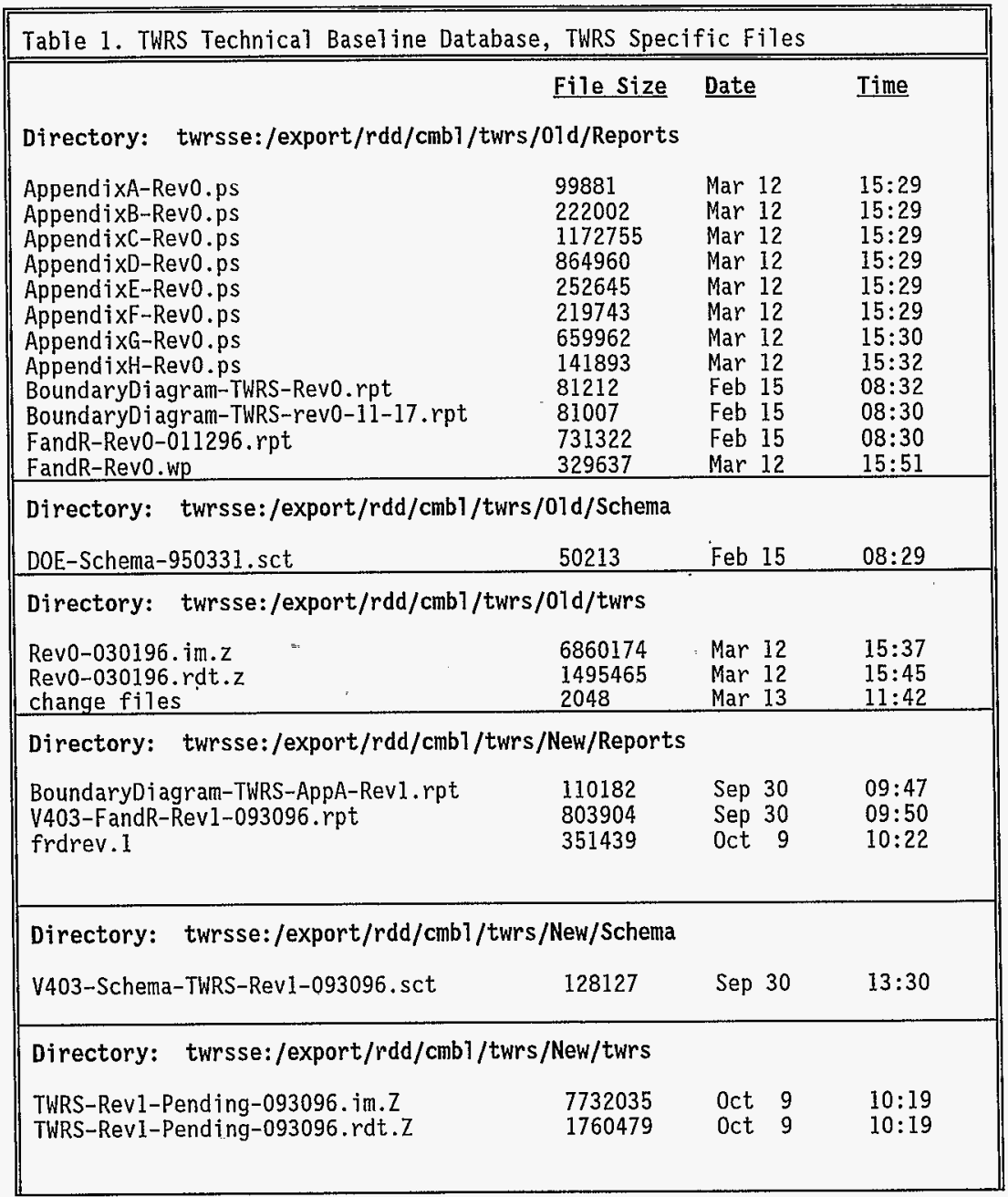


WHC-SD-WM-CSWD-079, Rev. 1

\begin{tabular}{|llll||}
\hline Table 1. TWRS Technical Baseline Database, TWRS Specific Files \\
\hline \hline Directory: twrsse:/export/rdd/cmb1/twrs/New/twrs/Documents & \\
AppA-Rev1-BoundaryDiagrams.ps & 135420 & 0ct 9 & $10: 14$ \\
AppB-Rev1.ps & 395974 & Oct 9 & $10: 14$ \\
AppC-Rev1.ps & 1979344 & Oct 9 & $10: 15$ \\
AppD-Rev1.ps & 1295649 & Oct 9 & $10: 15$ \\
AppE-Rev1.ps & 770033 & Oct 9 & $10: 15$ \\
AppF-Rev1.ps & 423249 & Oct 9 & $10: 15$ \\
AppG-Rev1.ps & 837187 & Oct 9 & $10: 16$ \\
AppH-Rev1.ps & 232623 & 0ct 9 & $10: 16$ \\
\hline
\end{tabular}


WHC-SD-WM-CSWD-079, Rev. 1

\begin{tabular}{|c|c|c|c|}
\hline & File Size & Date & Time \\
\hline \multicolumn{4}{|c|}{ Directory: twrsse:/export/rdd/cmbl/twrs/01d/change_files } \\
\hline TWR-95-112.rdt & 84750 & Feb & $13: 58$ \\
\hline TWR-95-113.rdt & 3798 & Feb 7 & $13: 59$ \\
\hline TWR-95-114.rdt & 52728 & Feb 7 & $13: 59$ \\
\hline TWR-95-116.rdt & 375556 & Feb 14 & $11: 39$ \\
\hline TWR-95-117.rdt & 488959 & Feb 7 & $14: 00$ \\
\hline TWR-95-118.rdt & 1620 & Feb 14 & $11: 45$ \\
\hline TWR-95-119.rdt & 33938 & Feb 14 & $11: 39$ \\
\hline TWR-95-120. rdt & 88405 & Feb 14 & $11: 39$ \\
\hline TWR-95-121.rdt & 18085 & Feb 14 & $11: 45$ \\
\hline TWR-95-122.rdt & 1510 & Feb 7 & $14: 04$ \\
\hline TWR-95-123.rdt & 32584 & Feb 14 & $11: 46$ \\
\hline TWR-95-124.rdt & 314063 & Feb 7 & $14: 08$ \\
\hline TWR-95-125.rdt & 2930 & Feb 14 & $11: 46$ \\
\hline TWR-95-126.rdt & 140027 & Feb 14 & $11: 46$ \\
\hline TWR-95-127.rdt & 170922 & Feb 14 & $11: 52$ \\
\hline TWR-95-128.rdt & 173908 & Feb 7 & $14: 25$ \\
\hline TWR-95-129.rdt & 243522 & Feb 14 & $11: 52$ \\
\hline TWR-95-130.rdt & 4790 & Feb 14 & $11: 53$ \\
\hline TWR-95-131.rdt & 6011 & Feb 14 & $11: 54$ \\
\hline TWR-95-132-133.rdt & 296535 & Feb 7 & $14: 07$ \\
\hline TWR-95-134.rdt & 192122 & Feb 14 & $11: 54$ \\
\hline TWR-95-135.rdt & 32959 & Feb 14 & $11: 54$ \\
\hline TWR-95-136. rdt & 53487 & Feb 14 & $11: 54$ \\
\hline TWR-95-137.rdt & 19801 & Feb 14 & $11: 55$ \\
\hline TWR-95-138.rdt & 19382 & Feb 14 & $11: 55$ \\
\hline TWR-95-139.rdt & 29777 & Feb 14 & $11: 55$ \\
\hline TWR-95-140.rdt & 17648 & Feb 14 & $11: 55$ \\
\hline TWR-95-141.rdt & 4408 & Feb 14 & $11: 56$ \\
\hline TWR-95-142.rdt & 100517 & Feb 14 & $11: 56$ \\
\hline TWR-95-143. rdt & 21795 & Feb 14 & $11: 56$ \\
\hline TWR-95-144.rdt & 53867 & Feb 7 & $14: 06$ \\
\hline TWR-95-145.rdt & 25188 & Feb 14 & $11: 56$ \\
\hline TWR-95-146.rdt & 40980 & Feb 7 & $14: 08$ \\
\hline TWR-95-147.rdt & 429665 & Feb 14 & $11: 57$ \\
\hline TWR-95-148A.rdt & 53781 & Feb 14 & $11: 33$ \\
\hline TWR-95-148B.rdt & 15136 & Feb 14 & $11: 33$ \\
\hline TWR-95-149.rdt & 28007 & Feb 7 & $14: 10$ \\
\hline TWR-96-200.rdt & 4069 & Feb 14 & $12: 01$ \\
\hline TWR-96-201.rdt & 19325 & Feb 14 & $12: 02$ \\
\hline TWR-96-202.rdt & 22259 & Feb 14 & $12: 02$ \\
\hline TWR-96-203.rdt & 19218 & Feb 14 & $12: 02$ \\
\hline TWR-96-204A.rdt & 46481 & Feb 14 & $11: 34$ \\
\hline TWR-96-204B.rdt & 7550 & Feb 14 & $11: 34$ \\
\hline TWR-96-205.rdt & 33599 & Feb 14 & $12: 02$ \\
\hline TWR-96-206.rdt & 8083 & Feb 14 & $12: 04$ \\
\hline TWR-96-207.rdt & 11928 & Feb 14 & $12: 04$ \\
\hline
\end{tabular}




\begin{tabular}{|c|c|c|c|}
\hline & File Size & Date & Time \\
\hline \multicolumn{4}{|c|}{ Directory: twrsse:/export/rdd/cmbl/twrs/01d/change_files (continued\} } \\
\hline TWR-96-208.rdt & 66652 & Feb 14 & $11: 05$ \\
\hline TWR-96-209A.rdt & 170712 & Feb 14 & $11: 34$ \\
\hline TWR-96-209B.rdt & 6076 & Feb 14 & $11: 34$ \\
\hline TWR-96-210.rdt & 22397 & Feb 14 & $11: 35$ \\
\hline TWR-96-211.rdt & 4252 & Feb 14 & $11: 35$ \\
\hline TWR-96-212.rdt & 176410 & Feb 14 & $11: 06$ \\
\hline TWR-96-213.rdt & 11659 & Feb 14 & $11: 06$ \\
\hline TWR-96-214.rdt & 20344 & Feb 14 & $11: 06$ \\
\hline TWR-96-215.rdt & 73283 & Feb 14 & $11: 06$ \\
\hline TWR-96-216.rdt & 154390 & Feb 14 & $11: 07$ \\
\hline TWR-96-218,rdt & 57608 & Feb 14 & $11: 07$ \\
\hline TWR-96-219.rdt & 108642 & Feb 14 & $11: 07$ \\
\hline TWR-96-220. rdt & 1893 & Feb 14 & $11: 07$ \\
\hline TWR-96-221.rdt & 45529 & Feb 14 & $11: 08$ \\
\hline TWR-96-222. rdt & 73363 & Feb 14 & $11: 08$ \\
\hline TWR-96-223.rdt & 30789 & Feb 14 & $11: 08$ \\
\hline TWR-96-224.rdt & 100380 & Feb 14 & $11: 08$ \\
\hline TWR-96-225.rdt & 134731 & Feb 14 & $11: 08$ \\
\hline TWR-96-241.rdt & 40456 & $\operatorname{Mar} 12$ & $15: 45$ \\
\hline
\end{tabular}




\begin{tabular}{|c|c|c|c|}
\hline & File Size & Date & Time \\
\hline \multicolumn{4}{|c|}{ Directory: twrsse:/export/rdd/cmbl/twrs/Delta } \\
\hline TWR-95-115.rdt & 259981 & Oct 9 & $10: 31$ \\
\hline TWR-96-217.rdt & 13688 & Sep 30 & $13: 08$ \\
\hline TWR-96-230.rdt & 12977 & Sep 30 & $13: 08$ \\
\hline TWR-96-23I.rdt & 10215 & Sep 30 & $13: 09$ \\
\hline TWR-96-232.rdt & 67373 & Sep 30 & $13: 06$ \\
\hline TWR-96-233.rdt & 78645 & Sep 30 & $13: 07$ \\
\hline TWR-96-235. rdt & 89617 & Sep 30 & $13: 22$ \\
\hline TWR-96-236.rdt & 82750 & Sep 30 & $13: 09$ \\
\hline TWR-96-238.rdt & 34515 & Sep 30 & $12: 58$ \\
\hline TWR-96-242.rdt & 10242 & Sep 30 & $13: 07$ \\
\hline TWR-96-244. rdt & 6830 & Sep 30 & $13: 09$ \\
\hline TWR-96-245.rdt & 7225 & Sep 30 & $13: 09$ \\
\hline TWR-96-246.rdt & 3166 & Sep 30 & $13: 09$ \\
\hline TWR-96-247.rdt & 12775 & Sep 30 & $13: 07$ \\
\hline TWR-96-248.rdt & 3118 & Sep 30 & $13: 10$ \\
\hline TWR-96-249+260.rdt & 14.37642 & Sep 30 & $12: 59$ \\
\hline TWR-96-250.rdt & 446758 & Sep 30 & $12: 59$ \\
\hline TWR-96-252.rdt & 97777 & Sep 30 & $13: 00$ \\
\hline TWR-96-254.rdt & 221037 & Sep 30 & $11: 35$ \\
\hline TWR-96-255.rdt & 1746 & Sep 30 & $13: 00$ \\
\hline TWR-96-259.rdt & 7071 & Sep 30 & $11: 35$ \\
\hline TWR-96-261.rdt & 71594 & Sep 30 & $11: 35$ \\
\hline TWR-96-263.rdt & 36175 & Sep 30 & $13: 01$ \\
\hline TWR-96-265.rdt & 44043 & Sep 30 & $11: 36$ \\
\hline TWR-96-267.rdt & 47317 & Sep 30 & $12: 57$ \\
\hline TWR-96-270.rdt & 232470 & Sep 30 & $11: 36$ \\
\hline TWR-96-271.rdt & 179065 & Sep 30 & $11: 36$ \\
\hline TWR-96-272.rdt & 37458 & Sep 30 & $11: 36$ \\
\hline TWR-96-274. rdt & 92465 & Sep 30 & $12: 58$ \\
\hline TWR-95-276.rdt & 7054 & Sep 30 & $11: 36$ \\
\hline TWR-96-277. rdt & 36466 & Sep 30 & $11: 37$ \\
\hline TWR-96-278. rdt & 283013 & Sep 30 & $11: 3 I$ \\
\hline TWR-96-279. rdt & 31119 & Sep 30 & $11: 37$ \\
\hline TWR-96-280. rdt & 9164 & Sep 30 & $11: 37$ \\
\hline TWR-96-281.rdt & 20255 & Sep 30 & $11: 37$ \\
\hline TWR-96-282.rdt & 65490 & $\operatorname{Sep} 30$ & $11: 38$ \\
\hline TWR-96-283.rdt & 289978 & Sep 30 & $11: 31$ \\
\hline TWR-96-284.rdt & 179777 & Sep 30 & $13: 42$ \\
\hline TWR-96-285. rdt & 208245 & Sep 30 & $11: 32$ \\
\hline W314-002.rdt & 42578 & Sep 30 & $13: 10$ \\
\hline W314-003.rdt & 19145 & Sep 30 & $13: 07$ \\
\hline W314-004.rdt & 342069 & Sep 30 & $13: 11$ \\
\hline
\end{tabular}




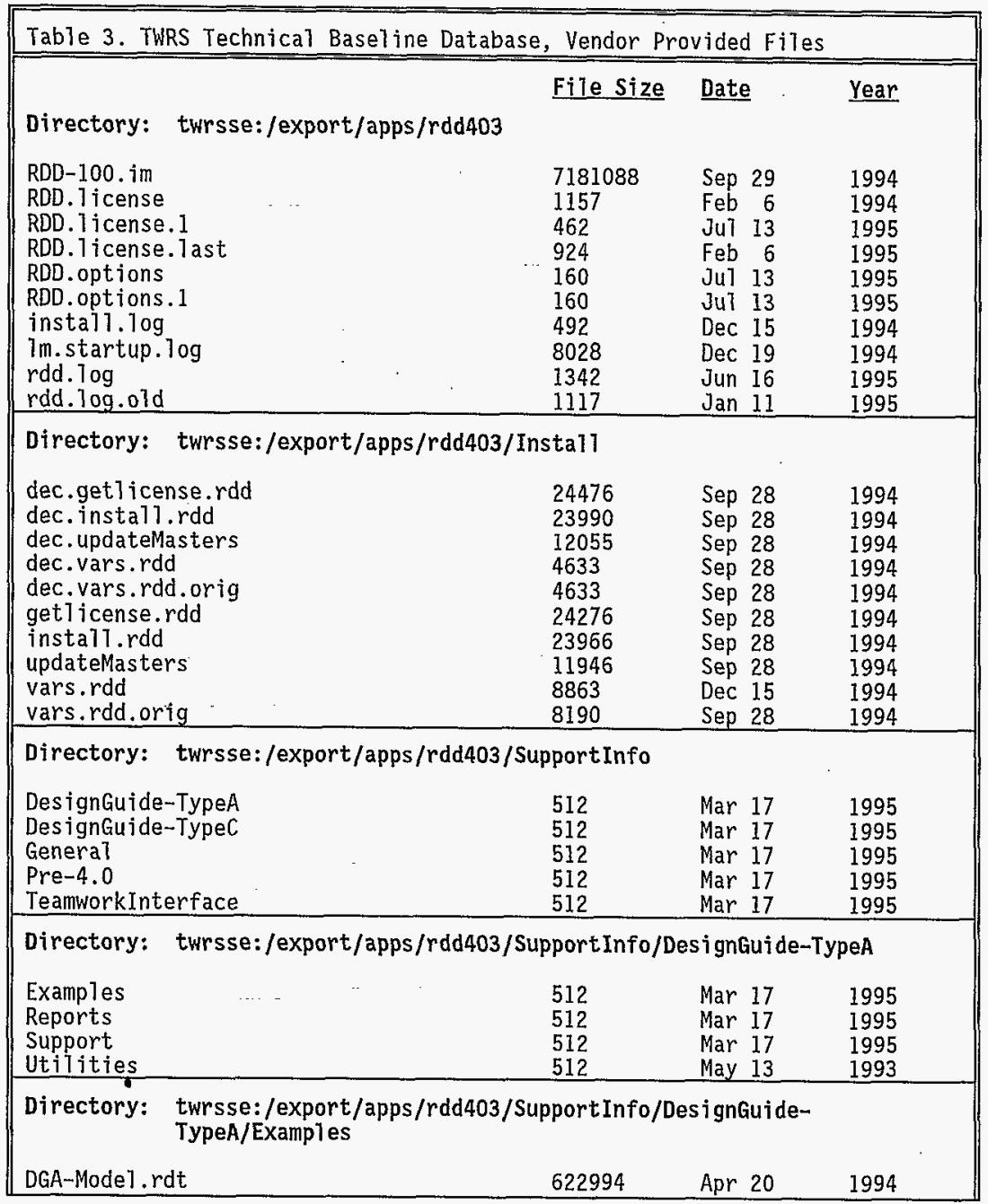




\begin{tabular}{|c|c|c|c|}
\hline & File size & Date & Year \\
\hline \multicolumn{4}{|c|}{$\begin{array}{l}\text { Directory: } \begin{array}{l}\text { twrsse:/export/apps/rdd403/SupportInfo/DesignGuide- } \\
\text { TypeA/Reports }\end{array}\end{array}$} \\
\hline $\begin{array}{l}\text { DGA-B1Categories.rdt } \\
\text { DGA-BIDoc.rdt } \\
\text { DGA-B2Categories.rdt } \\
\text { DGA-B2Doc.rdt } \\
\text { DGA-IRSCategories.rdt } \\
\text { DGA-IRSDoc.rdt } \\
\text { DGA-ProjectUniqueID.rpt } \\
\text { DGA-Reports.rpt } \\
\text { DGA-SRSCategories.rdt } \\
\text { DGA-SRSDoc.rdt } \\
\text { DGA-SSDDCategories.rdt } \\
\text { DGA-SSDDDoc.rdt } \\
\text { DGA-SSS-ASpecDoc.rdt } \\
\text { DGA-SSSCategories.rdt }\end{array}$ & $\begin{array}{l}3813 \\
17139 \\
3285 \\
14247 \\
660 \\
4459 \\
27106 \\
1215285 \\
2676 \\
13010 \\
3264 \\
16207 \\
19061 \\
6018 \\
\end{array}$ & $\begin{array}{lr}\text { Aug } & 30 \\
\text { Aug } & 30 \\
\text { Aug } & 30 \\
\text { Aug } & 30 \\
\text { Aug } & 30 \\
\text { Aug } & 30 \\
\text { Mar } & 9 \\
\text { Sep } & 6 \\
\text { Apr } & 11 \\
\text { Apr } & 13 \\
\text { Jan } & 25 \\
\text { Aug } & 30 \\
\text { Aug } & 30 \\
\text { Apr } & 11 \\
\end{array}$ & $\begin{array}{l}1994 \\
1994 \\
1994 \\
1994 \\
1994 \\
1994 \\
1994 \\
1994 \\
1994 \\
1994 \\
1994 \\
1994 \\
1994 \\
1994\end{array}$ \\
\hline \multicolumn{4}{|c|}{$\begin{array}{l}\text { Directory: twrsse:/export/apps/rdd403/SupportInfo/DesignGuide- } \\
\text { TypeA/Support }\end{array}$} \\
\hline $\begin{array}{l}\text { DG-MUMQueries.rqt } \\
\text { DGA-Cons istencyChecks,cct } \\
\text { DGA-MEVTemplates.tpt } \\
\text { DGA-Schema.sct }\end{array}$ & $\begin{array}{l}342583 \\
86491 \\
15873 \\
72670 \\
\end{array}$ & $\begin{array}{lr}\text { Mar } 22 \\
\text { Mar } 31 \\
\text { Aug } 2 \\
\text { Sep } 8 \\
\end{array}$ & $\begin{array}{l}1994 \\
1994 \\
1994 \\
1994 \\
\end{array}$ \\
\hline \multicolumn{4}{|c|}{ Directory: twrsse:/export/apps/rdd403/SupportInfo/DesignGuide-TypeC } \\
\hline $\begin{array}{l}\text { Examples } \\
\text { Reports } \\
\text { Support } \\
\text { Utilities } \\
\end{array}$ & $\begin{array}{l}512 \\
512 \\
512 \\
512 \\
\end{array}$ & $\begin{array}{l}\text { Mar } 17 \\
\text { Mar } 17 \\
\text { Mar } 17 \\
\text { May } 13\end{array}$ & $\begin{array}{l}1995 \\
1995 \\
1995 \\
1993 \\
\end{array}$ \\
\hline \multicolumn{4}{|c|}{$\begin{array}{l}\text { Directory: twrsse:/export/apps/rdd403/SupportInfo/DesignGuide- } \\
\text { TypeC/Examples }\end{array}$} \\
\hline DGC-Model.rdt & 905127 & Apr 20 & 1994 \\
\hline \multicolumn{4}{|c|}{ 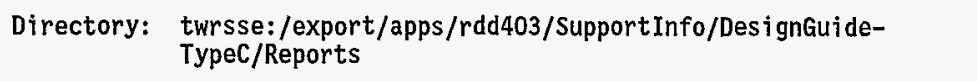 } \\
\hline $\begin{array}{l}\text { DGC-ProjectUniqueID.rpt } \\
\text { DGC-Reports.rpt } \\
\text { DGC-SSDDCategories.rdt } \\
\text { DGC-SSDDDoc.rdt } \\
\text { DGC-SSS-ASpecDoc.rdt } \\
\text { DGC-SSSCategories.rdt }\end{array}$ & $\begin{array}{l}28929 \\
1091441 \\
2275 \\
12110 \\
19116 \\
5998\end{array}$ & $\begin{array}{lr}\text { May } & 6 \\
\text { Aug } & 30 \\
\text { Mar } 15 \\
\text { Aug } & 30 \\
\text { Aug } & 30 \\
\text { Mar } 15\end{array}$ & $\begin{array}{l}1994 \\
1994 \\
1994 \\
1994 \\
1994 \\
1994\end{array}$ \\
\hline
\end{tabular}




\begin{tabular}{|c|c|c|c|}
\hline & File Size & Date & Year \\
\hline \multicolumn{4}{|c|}{$\begin{array}{l}\text { Directory: twrsse:/export/apps/rdd403/SupportInfo/DesignGuide- } \\
\text { Typec/Support }\end{array}$} \\
\hline $\begin{array}{l}\text { DG-MUMQueries.rqt } \\
\text { DGC-ConsistencyChecks.cct } \\
\text { DGC-MEVTemplates.tpt } \\
\text { DGC-Schema.sct }\end{array}$ & $\begin{array}{l}390092 \\
143177 \\
4611 \\
71515 \\
\end{array}$ & $\begin{array}{lr}\text { May } & 2 \\
\text { Dec } & 13 \\
\text { Aug } & 2 \\
\text { Sep } & 8 \\
\end{array}$ & $\begin{array}{l}1994 \\
1993 \\
1994 \\
1994 \\
\end{array}$ \\
\hline \multicolumn{4}{|c|}{ Directory: twrsse:/export/apps/rdd403/SupportInfo/Genera1 } \\
\hline $\begin{array}{l}\text { Examples } \\
\text { Reports } \\
\text { Support } \\
\text { Utilities } \\
\end{array}$ & $\begin{array}{l}512 \\
512 \\
512 \\
1024 \\
\end{array}$ & $\begin{array}{l}\text { Mar } 17 \\
\text { Mar } 17 \\
\text { Mar } 17 \\
\text { Mar } 17\end{array}$ & $\begin{array}{l}1995 \\
1995 \\
1995 \\
1995 \\
\end{array}$ \\
\hline \multicolumn{4}{|c|}{ Directory: twrsse:/export/apps/rdd403/SupportInfo/General/Examples } \\
\hline $\begin{array}{l}\text { ATCexample.rdt } \\
\text { SATCS Draft Specs } \\
\text { Tutorials.rdt }\end{array}$ & $\begin{array}{l}61368 \\
2824 \\
304626 \\
\end{array}$ & $\begin{array}{lr}\text { Dec } & 2 \\
\text { Jun } & 14 \\
\text { Dec } & 13 \\
\end{array}$ & $\begin{array}{l}1993 \\
1993 \\
1993 \\
\end{array}$ \\
\hline \multicolumn{4}{|c|}{ Directory: twrsse:/export/apps/rdd403/SupportInfo/General/Reports } \\
\hline $\begin{array}{l}\text { ElementDescriptionReport.rpt } \\
\text { OwnershipReport.rpt } \\
\text { SEN-Sections.rdt } \\
\text { SchemaDescriptionReport.rpt } \\
\text { SystemEngineeringNotebook.rpt }\end{array}$ & $\begin{array}{l}33256 \\
44542 \\
10111 \\
40884 \\
254552 \\
\end{array}$ & $\begin{array}{lr}\text { Mar } & 29 \\
\text { May } & 9 \\
\text { May } & 9 \\
\text { Apr } & 13 \\
\text { Sep } & 27 \\
\end{array}$ & $\begin{array}{l}1994 \\
1994 \\
1994 \\
1994 \\
1994 \\
\end{array}$ \\
\hline \multicolumn{4}{|c|}{ Directory: twrsse:/export/apps/rdd403/SupportInfo/General/Support } \\
\hline $\begin{array}{l}\text { ConsistencyChecks.cct } \\
\text { MUMQueries.rqt } \\
\text { StandardTemplates.tpt }\end{array}$ & $\begin{array}{l}87971 \\
283929 \\
15878\end{array}$ & $\begin{array}{lr}\text { Aug } & 2 \\
\text { Sep } & 28 \\
\text { Aug } & 16\end{array}$ & $\begin{array}{l}1994 \\
1994 \\
1994\end{array}$ \\
\hline
\end{tabular}




\begin{tabular}{|c|c|c|c|}
\hline & File size & Date & Year \\
\hline \multicolumn{4}{|c|}{ Directory: twrsse:/export/apps/rdd403/SupportInfo/General/Utilities } \\
\hline $\begin{array}{l}\text { AllocationBuilder.rpt } \\
\text { ChangeType.rpt } \\
\text { ComputePerformanceIndexes.rpt } \\
\text { DatabaseProfile.rpt } \\
\text { EraseExtraCRs.rpt } \\
\text { FindAndReplace.rpt } \\
\text { FindZeroFooterSize.rpt } \\
\text { FunctionHierBuilder.rpt } \\
\text { GhostBuster.rpt } \\
\text { GhostFinder.rpt } \\
\text { ItemLevels.rpt } \\
\text { ListReportMultiColumns.rpt } \\
\text { ListSelectNodeAnnotations.rpt } \\
\text { ListSortKeyProblems.rpt } \\
\text { PrintUserSelectedAttributes.rpt } \\
\text { ReAuthorElements.rpt } \\
\text { ReNumberHierarchy.rpt } \\
\text { RemoveCarriageReturns.rpt } \\
\text { ReportHierarchy.rat } \\
\text { SchemaChanges.rpt } \\
\text { SourceParser.rpt } \\
\text { VariantCompare.rpt } \\
\text { VersionCompare.rpt }\end{array}$ & $\begin{array}{l}49623 \\
26863 \\
9480 \\
52066 \\
4127 \\
29659 \\
4496 \\
26831 \\
1973 \\
2168 \\
29226 \\
3018 \\
9223 \\
8862 \\
20500 \\
6956 \\
20909 \\
28304 \\
10369 \\
55832 \\
27594 \\
75401 \\
90534\end{array}$ & $\begin{array}{lr}\text { Jun } & 6 \\
\text { Jun } & 6 \\
\text { May } & 11 \\
\text { Mar } & 29 \\
\text { Sep } & 4 \\
\text { Mar } & 29 \\
\text { Mar } & 25 \\
\text { Oct } & 6 \\
\text { Sep } & 4 \\
\text { Sep } & 4 \\
\text { Mar } & 29 \\
\text { Mar } & 25 \\
\text { Mar } & 25 \\
\text { Mar } & 25 \\
\text { Mar } & 25 \\
\text { Mar } & 29 \\
\text { Apr } & 20 \\
\text { Jul } & 15 \\
\text { Mar } & 25 \\
\text { Mar } & 29 \\
\text { Aug } & 16 \\
\text { Aug } & 29 \\
\text { May } & 6\end{array}$ & $\begin{array}{l}1994 \\
1994 \\
1994 \\
1994 \\
1993 \\
1994 \\
1994 \\
1993 \\
1993 \\
1993 \\
1994 \\
1994 \\
1994 \\
1994 \\
1994 \\
1994 \\
1994 \\
1994 \\
1994 \\
1994 \\
1994 \\
1994 \\
1994\end{array}$ \\
\hline \multicolumn{4}{|c|}{ Directory: twrsse:/export/apps/rdd403/SupportInfo/Pre-4.0 } \\
\hline $\begin{array}{l}\text { Examples } \\
\text { Reports } \\
\text { Support } \\
\text { Utilities }\end{array}$ & $\begin{array}{l}512 \\
512 \\
512 \\
512 \\
\end{array}$ & $\begin{array}{ll}\text { Aug } & 5 \\
\text { Mar } & 17 \\
\text { Mar } 17 \\
\text { Mar } 17\end{array}$ & $\begin{array}{l}1993 \\
1995 \\
1995 \\
1995\end{array}$ \\
\hline \multicolumn{4}{|c|}{ Directory: twrsse:/export/apps/rdd403/SupportInfo/Pre-4.0/Reports } \\
\hline $\begin{array}{l}\text { DIDtemplate.rdt } \\
\text { IRSConsistencies.rpt } \\
\text { IRSdocumentElements.rdt } \\
\text { InterfaceRequirementsSpec.rpt } \\
\text { RequirementsAl location.rpt } \\
\text { RequirementsTraceability.rpt } \\
\text { SENdocumentE] ements.rdt } \\
\text { SSDDConsistencies.rpt } \\
\text { SSDDdocumentElements.rdt } \\
\text { SSSConsistencies.rpt } \\
\text { SSSdocumentElements.rdt } \\
\text { SystemEngineeringNotebook.rpt } \\
\text { SystemSegmentDesignDoc.rpt } \\
\text { SystemSegmentSpecification.rpt }\end{array}$ & $\begin{array}{l}78880 \\
87821 \\
6031 \\
123993 \\
94380 \\
69677 \\
5032 \\
121910 \\
19974 \\
118905 \\
36290 \\
312040 \\
214720 \\
179412\end{array}$ & $\begin{array}{ll}\text { Sep } & 8 \\
\text { Sep } & 8 \\
\text { Sep } & 8 \\
\text { Sep } & 9 \\
\text { Sep } & 8 \\
\text { Sep } & 8 \\
\text { Sep } & 8 \\
\text { Sep } & 8 \\
\text { Sep } & 8 \\
\text { Sep } & 8 \\
\text { Sep } & 8 \\
\text { Sep } & 4 \\
\text { Sep } & 8 \\
\text { Sep } & 9\end{array}$ & $\begin{array}{l}1993 \\
1993 \\
1993 \\
1993 \\
1993 \\
1993 \\
1993 \\
1993 \\
1993 \\
1993 \\
1993 \\
1993 \\
1993 \\
1993\end{array}$ \\
\hline
\end{tabular}




\begin{tabular}{|c|c|c|c|}
\hline & File Size & Date & Year \\
\hline \multicolumn{4}{|c|}{ Directory: twrsse:/export/apps/rdd403/SupportInfo/Pre-4.0/Support } \\
\hline \multicolumn{4}{|c|}{ Directory: twrsse:/export/apps/rdd403/SupportInfo/Pre-4.0/Uti1ities } \\
\hline $\begin{array}{l}\text { Directory: } \text { twrsse:/exp } \\
\text { Reports } \\
\text { Utilities } \\
\end{array}$ & $\begin{array}{l}\text { pportInfo/T } \\
512 \\
512 \\
\end{array}$ & $\begin{array}{l}\text { amworkInt } \\
\text { Mar } 17 \\
\text { Mar } 17\end{array}$ & $\begin{array}{l}\text { aces } \\
1995 \\
1995 \\
\end{array}$ \\
\hline \multicolumn{4}{|c|}{$\begin{array}{l}\text { Directory: } \begin{array}{l}\text { twrsse:/export/apps/rdd403/SupportInfo/TeamworkInterfaces/ } \\
\text { Reports }\end{array}\end{array}$} \\
\hline DataDictionary.rpt & 47148 & Jul 20 & 1993 \\
\hline \multicolumn{4}{|c|}{$\begin{array}{l}\text { Directory: twrsse:/export/apps/rdd403/SupportInfo/TeamworkInterfaces/ } \\
\text { Utilities }\end{array}$} \\
\hline $\begin{array}{l}\text { RDD. Cadre config } \\
\text { RDD.Cadre-menu } \\
\text { RDD.Cadre prog }\end{array}$ & $\begin{array}{l}348 \\
961 \\
49152 \\
\end{array}$ & $\begin{array}{ll}\text { Dec } & 15 \\
\text { Dec } & 15 \\
\text { Sep } & 23 \\
\end{array}$ & $\begin{array}{l}1994 \\
1994 \\
1992 \\
\end{array}$ \\
\hline \multicolumn{4}{|c|}{ Directory: twrsse:/export/apps/bin/rdd403/bin } \\
\hline $\begin{array}{l}\text { dec. } 1 \mathrm{~m} . \text { startup } \\
\text { dec.rdd } 4.0 .3 \\
\text { 1m.startup } \\
\text { mips } \\
\text { rdd403 } \rightarrow \text { rdd } 4 \cdot 0.3 \\
\text { rdd } 4.0 .3 \\
\text { sun4 } \\
\text { sun4-SOL } \\
\text { vars.rdd }\end{array}$ & $\begin{array}{l}8954 \\
15067 \\
8881 \\
512 \\
9 \\
15079 \\
512 \\
512 \\
8863 \\
\end{array}$ & $\begin{array}{lr}\text { Sep } & 28 \\
\text { Sep } & 28 \\
\text { Sep } & 28 \\
\text { Mar } & 17 \\
\text { Mar } & 17 \\
\text { Sep } & 28 \\
\text { Mar } & 17 \\
\text { Jut } & 7 \\
\text { Dec } & 15 \\
\end{array}$ & $\begin{array}{l}1994 \\
1994 \\
1994 \\
1995 \\
1995 \\
1994 \\
1995 \\
1995 \\
1994 \\
\end{array}$ \\
\hline \multicolumn{4}{|c|}{ Directory: twrsse:/export/apps/rdd403/bin/mips } \\
\hline $\begin{array}{l}\text { ALC fiex } \\
\text { RDD } 100 \\
\text { Tmdown } \\
\text { Imgrd } \\
\text { Imhostid } \\
\text { imremove } \\
\text { Tmreread } \\
\text { imstat } \\
\end{array}$ & $\begin{array}{l}331776 \\
1075440 \\
353024 \\
270336 \\
353024 \\
353024 \\
353024 \\
353024 \\
\end{array}$ & $\begin{array}{lr}\text { Aug } & 29 \\
\text { Sep } & 8 \\
\text { Sep } & 2 \\
\text { Jun } & 13 \\
\text { Sep } & 2 \\
\text { Sep } & 2 \\
\text { Sep } & 2 \\
\text { Sep } & 2 \\
\end{array}$ & $\begin{array}{l}1994 \\
1994 \\
1994 \\
1994 \\
1994 \\
1994 \\
1994 \\
1994 \\
\end{array}$ \\
\hline
\end{tabular}




\begin{tabular}{|c|c|c|c|}
\hline & File size & Date & Year \\
\hline \multicolumn{4}{|c|}{ Directory: twrsse:/export/apps/rdd403/bin/sun4 } \\
\hline $\begin{array}{l}\text { ALC flex } \\
\text { RDD-100 } \\
\text { Imdown } \\
\text { Tmgrd } \\
\text { Tmhostid } \\
\text { Tmremove } \\
\text { Imreread } \\
\text { Tmstat } \\
\end{array}$ & $\begin{array}{l}262144 \\
720896 \\
155648 \\
188416 \\
155648 \\
155648 \\
155648 \\
155648 \\
\end{array}$ & $\begin{array}{lr}\text { Aug } & 29 \\
\text { Sep } & 8 \\
\text { May } & 24 \\
\text { Jun } & 9 \\
\text { May } & 24 \\
\text { May } & 24 \\
\text { May } & 24 \\
\text { May } & 24 \\
\end{array}$ & $\begin{array}{l}1994 \\
1994 \\
1994 \\
1994 \\
1994 \\
1994 \\
1994 \\
1994 \\
\end{array}$ \\
\hline \multicolumn{4}{|c|}{ Directory: twrsse:/export/bin/apps/rdd403/sun4-SOL } \\
\hline $\begin{array}{l}\text { ALC flex } \\
\text { RDD-100 } \\
\text { RDD-100.orig } \\
\text { Tmdown } \\
\text { lmgrd } \\
\text { Imhostid } \\
\text { Tmremove } \\
\text { Imreread } \\
\text { Imstat }\end{array}$ & $\begin{array}{l}308028 \\
964708 \\
838580 \\
181436 \\
229088 \\
181436 \\
181436 \\
181436 \\
181436 \\
\end{array}$ & $\begin{array}{lr}\text { Aug } & 29 \\
\text { Ju1 } & 7 \\
\text { Sep } & 8 \\
\text { May } & 24 \\
\text { Jun } & 9 \\
\text { May } & 24 \\
\text { May } & 24 \\
\text { May } & 24 \\
\text { May } & 24 \\
\end{array}$ & $\begin{array}{l}1994 \\
1995 \\
1994 \\
1994 \\
1994 \\
1994 \\
1994 \\
1994 \\
1994\end{array}$ \\
\hline
\end{tabular}

\begin{tabular}{|c|c|}
\hline Type of computer: & Sun SPARC Server \\
\hline Type of CPU: & Server 1000 \\
\hline Operating system: & SOLARIS 2.3 \\
\hline Minimum memory: & 40 Megabytes \\
\hline $\begin{array}{l}\text { Minimum hard drive } \\
\text { size: }\end{array}$ & 1 Gigabyte \\
\hline Network: & Hanford HLAN \\
\hline Tape Drive: & $4 \mathrm{~mm}$ tape drive \\
\hline 0ther software needs: & Newsprint software program is used for printing \\
\hline
\end{tabular}

